\title{
19. Frankfurter Interdisziplinäres Symposium für Innovative Diagnostik und Therapie - FISI 2018
}

Sehr geehrte Kollegin, sehr geehrter Kollege,

es ist uns eine große Freude, Sie auch in diesem Jahr zum 19. FISI-Symposium mit dem Titel „MRI and beyond“ am 14. und 15. September 2018 in Frankfurt am Main einzuladen.

In der täglichen Routine bedeutet der interdisziplinäre Austausch mit anderen Fachdisziplinen eine essenzielle Basis der radiologischen Diagnostik. Traditionell versteht sich das FISI in diesem Sinne als interdisziplinäre Veranstaltung mit dem Ziel, radiologisches Arbeiten im klinischen Kontext zu beleuchten. Mit dem Motto „MRI and beyond“ wollen wir über den Tellerrand blicken und dabei Etabliertes hinterfragen und neue Innovationen kritisch diskutieren. Als wichtige Partner stehen uns dabei klinische Kollegen zu den Themenschwerpunkten Kopf-/ Hals-Diagnostik, kardiale Diagnostik, muskuloskelettale Diagnostik, gynäkologische und abdominelle Diagnostik zur Seite.

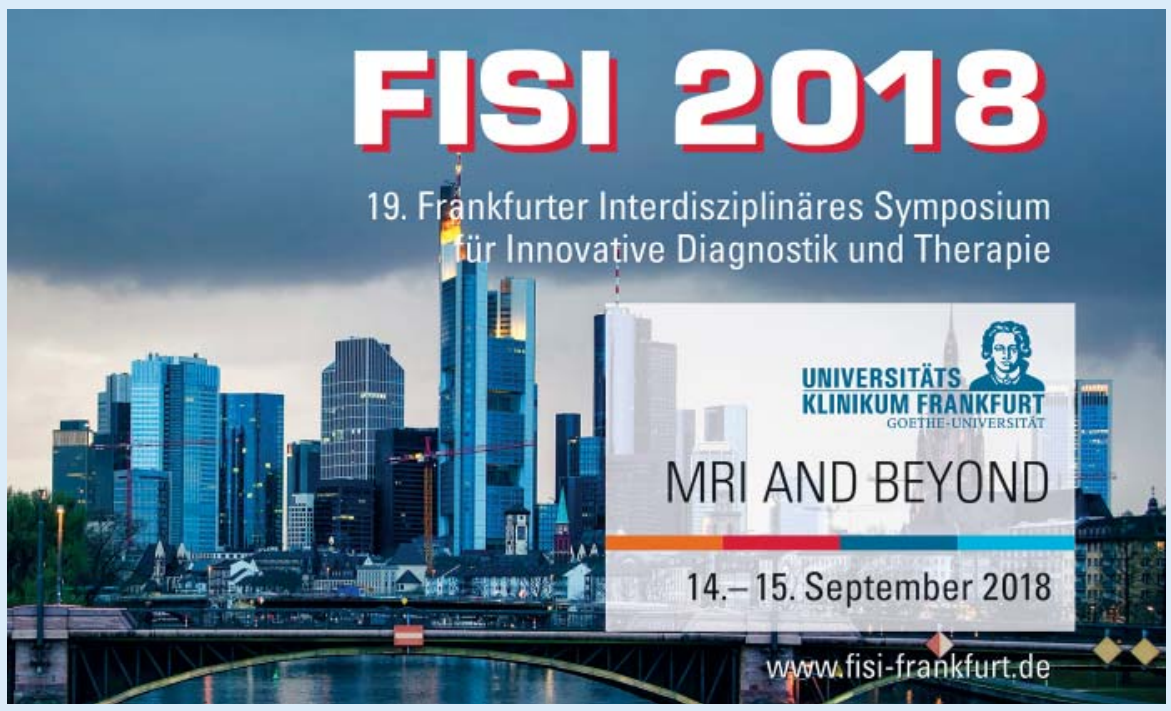

In einem neuroradiologischen Themenkomplex am Freitag wird sich Prof. Dr. Michael Forsting aus Essen mit dem Thema „Von 7 Tesla bis zur Artificial Intelligence“ beschäftigen und uns in die Zukunft neuroradiologsicher Diagnostik blicken lassen.
Prof. Dr. Alexander Radbruch aus Essen wird zu dem weiterhin aktuellen Thema der zerebralen Gadolinium-Ablagerungen sprechen, um uns auf den aktuellen Stand der Forschung zu bringen. In einer anschließenden Session werden u. a. Prof. Dr. Michael 
Lell aus Erlangen über die TNM-Klassifikation im Kopf-Hals-Bereich und Frau Prof. Dr. Gabriele Krombach aus Gießen über die MRT-Diagnostik der Speicheldrüsen referieren. Bei einem anschließenden Get-Together wird Gelegenheit sein, interessante Themen weiter zu diskutieren.

In der ersten Session am Samstagvormittag referiert Prof. Dr. Eike Nagel als einer der weltweit führenden Spezialisten auf dem Gebiet der kardialen Bildgebung über „Quantifizierung, Strukturierung und Validierung der kardialen MRT“. In einer zweiten Session am Vormittag über muskuloskelettale Bildgebung unter dem Vorsitz von Prof. Dr. Tim Meyer, Teamarzt der deutschen Fußballnationalmannschaft und Professor für Sport- und Präventivmedizin an der Universität des Saarlandes, werden u. a. Prof. Dr. Marc-André Weber aus Rostock über die MRT des Kniegelenks und Frau Prof. Dr. Katrin Eichler aus Frankfurt über die MRT bei Muskelverletzungen sprechen.

In einem gynäkologischen Block am Nachmittag kommen Prof. Dr. Markus MüllerSchimpfle aus Frankfurt, Leiter der AG Mammadiagnostik der DRG, sowie Prof. Dr. Rüdiger Schulz-Wendtland aus Erlangen, Präsident der deutschen Gesellschaft für Senologie, zu Wort, bevor sich der letzte Themenblock über abdominelle und urologische Bildgebung anschließt. Prof. Dr. Andreas Schreyer aus Regensburg, stellvertretender Vorsitzender der AG Gastrointestinal- und Abdominaldiagnostik, wird hier zur strukturierten Befundung des Pankreas vortragen und klärt die Frage, was der Kliniker vom Radiologen wissen möchte.

Wir hoffen, auch dieses Jahr einen für Sie interessanten Kongress zusammengestellt zu haben und würden uns sehr freuen, Sie am 14. und 15. September zum FISI-Symposium in Frankfurt begrüßen zu dürfen.

Thomas ]. Vogl

Renate Hammerstingl

Benjamin Kaltenbach

\section{Online-Registrierung und aktuelle Infor-} mationen

www.fisi-frankfurt.de

\section{Veranstaltungsort}

Universitätsklinikum Frankfurt Vorlesungs- und Kongressbereich Theodor-Stern-Kai 7, 60590 Frankfurt

\section{Kongressorganisation}

KelCon $\mathrm{GmbH}$, Cathleen Raum

Liebigstraße 16, 63500 Seligenstadt

Tel.: + 49 (0) $6182-9466617$

Fax: + $49(0) 6182-9466644$

\section{Wissenschaftliche Leitung}

Prof. Dr. Thomas J. Vogl

Dr. med. Renate Hammerstingl

Dr. med. Benjamin Kaltenbach 